\section{A Case of Antibiotic-Induced Posterior Reversible Encephalopathy Syndrome}

\author{
Brian Wesley Gilbert' ${ }^{1}$, Ali Gabriel', and Laura Velazquez'
}

Hospital Pharmacy

2020, Vol. 55(5) 338-34I

(c) The Author(s) 2019

Article reuse guidelines:

sagepub.com/journals-permissions DOI: 10.1 I 77/ 0018578719844706 journals.sagepub.com/home/hpx

(S)SAGE

\begin{abstract}
Purpose: To report a case of posterior reversible encephalopathy syndrome (PRES) in a 75 year-old patient who was taking concomitant ciprofloxacin and metronidazole. Method: Case report Results: A patient had been prescribed ciprofloxacin and metronidazole during a recent hospitalization and continued this regimen outpatient. Two weeks after discharge and 3 weeks after initiation of her regimen, she was brought to the emergency department after developing acute weakness and lightheadedness. After admission, the patient declined more rapidly and began seizing with subsequent intubation. Initial computed tomographic (CT) imaging showed no acute neurological abnormalities, and a sepsis workup was initiated. After negative CT, a magnetic resonance imaging scan was performed that showed a T2 flair and hyperdensity consistent with PRES. The final diagnosis was considered to be PRES secondary to ciprofloxacin/metronidazole utilization. Conclusion: Antibiotic induced PRES is a condition that needs to be explored more thoroughly.
\end{abstract}

\title{
Keywords
}

adverse drug reactions, adverse drug reactions reporting/monitoring, critical care, infectious diseases

Posterior reversible encephalopathy syndrome (PRES) is a neurological syndrome that is associated with nonspecific symptoms, including headache, confusion, visual disturbances, myoclonus, seizures, and impaired consciousness. While the pathogenesis is not completely understood, there have been increasing reports, with associated risk factors identified as hypertension, renal dysfunction, eclampsia, sepsis, and some medications. ${ }^{1}$ Immunosuppressants, linezolid, duloxetine, and ciprofloxacin have all had cases reported of this phenomenon. ${ }^{2-5}$ The PRES is associated with lesions that may or may not be symmetrical and can be seen on magnetic resonance imaging (MRI) in the posterior parieto-occipital regions.

Metronidazole-induced encephalopathy (MIE) is a disorder associated with metronidazole use and presents with altered mental status, aphasia, dysarthria, and, in some instances, seizures. ${ }^{6-8}$ Prolonged exposure to metronidazole has been identified as the most likely cause and is associated with unique neuroimaging such as symmetric bilateral fluidattenuated inversion recovery (FLAIR) seen on MRI at the dentate nuclei, dorsal medulla, pons, or midbrain regions.

While there have been numerous documented cases of MIE in patients on chronic metronidazole therapy, the authors' literature search did not return any documented cases of metronidazole-induced PRES, and there has only been one case of ciprofloxacin-induced PRES in the literature to date. ${ }^{2}$ Here, we present a case of a patient on longterm ciprofloxacin and metronidazole therapy who developed PRES. The concomitant utilization of each agent and the temporal relationship of discontinuation make this a bit of an enigma.

\section{Case Description}

A 75-year-old woman with a history of hypertension, anemia, hypothyroidism, and recent resolving abdominal abscess with diverticulitis requiring treatment with ciprofloxacin and metronidazole (due to penicillin and cephalosporin allergies; both anaphylaxis) presented to the emergency department (ED) from a rehabilitation facility after increased somnolence, fatigue, nausea/vomiting, and confusion. The planned treatment duration with ciprofloxacin and metronidazole was 4 weeks after the last incision and drainage. After performing a computed tomography (CT) of the abdomen to rule out an abdominal abscess, the patient began to experience seizure-like activity and was subsequently intubated to ensure airway protection. The patient was hypotensive and euglycemic before and after intubation. There were no initial laboratory abnormalities, except a small serum creatinine elevation from 1.6 to $1.8 \mathrm{mg} / \mathrm{dL}$ (141.4-159.1 $\mu \mathrm{mol} / \mathrm{L})$. After discussion with family, it was found that the patient had no prior history of seizures. After

\footnotetext{
'Wesley Medical Center, Wichita, KS, USA
}

Corresponding Author:

Brian Wesley Gilbert, Wesley Medical Center, 550 N Hillside Ave, Wichita, KS 67214, USA.

Email: brian.gilbert.pharmd@gmail.com 


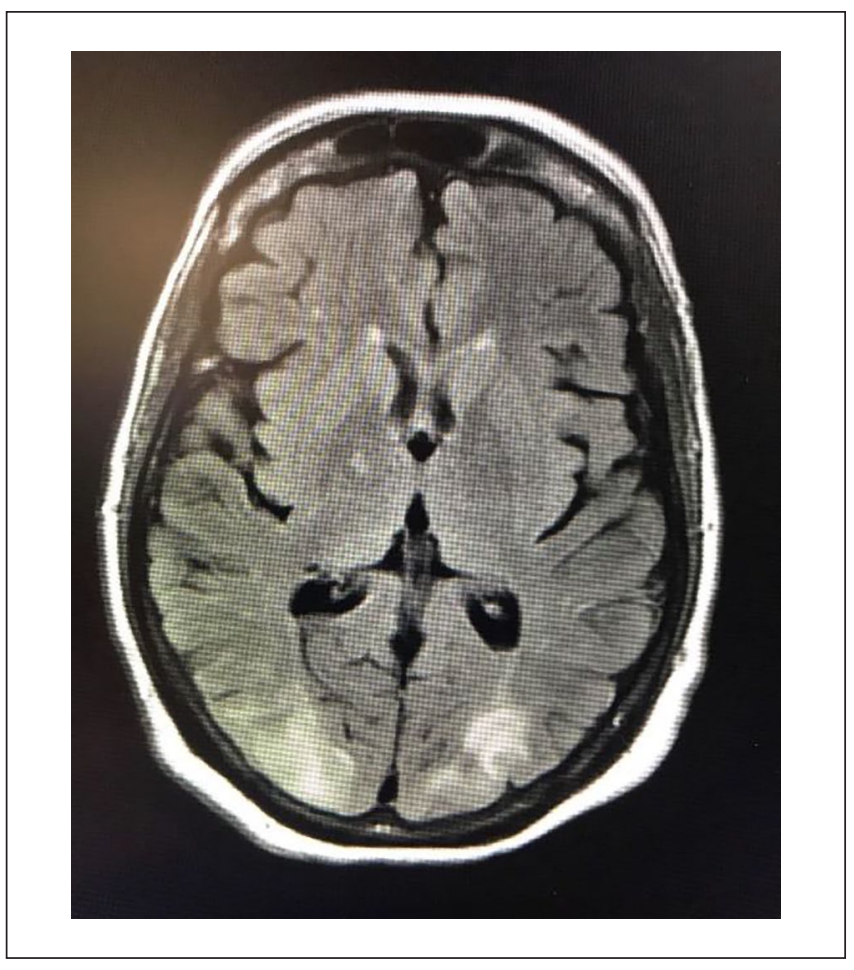

Figure I. Magnetic resonance imaging of brain without contrast showing T2 fluid-attenuated inversion recovery in parietooccipital region.

the seizure and intubation, the patient was given a loading dose of $1000 \mathrm{mg}$ of levetiracetam, and no further seizures were noted. During the patient's course in the ED, a medication reconciliation was performed, and it was found that the patient had been on ciprofloxacin $500 \mathrm{mg}$ twice daily and metronidazole $500 \mathrm{mg}$ twice daily for the past 3 weeks. After intubation, a stat MRI (Figure 1) of the brain was performed, which showed bilateral symmetric areas of increased T2/ FLAIR signal intensity in the deep subcortical white matter of the parietal occipital regions, which was suggested to represent PRES. During day 1, the patient was transferred to the medical intensive care unit. As the patient still required antibiotic treatment, as determined by surgery and infectious diseases teams, the decision was made to continue metronidazole, transition from ciprofloxacin to aztreonam, and add vancomycin empirically, with a workup for sepsis initiated. Initial signs pointing toward sepsis included hypotension and leukocytosis despite prior abscess treatment. On day 2 of admission, the patient remained intubated, had an increase in serum creatinine to $4.0 \mathrm{mg} / \mathrm{dL}(353.6 \mu \mathrm{mol} / \mathrm{L})$, required vasopressor support, and blood cultures were negative to date. No other culture data or microbiology was done. On day 3, a continuous electroencephalogram was performed, which showed diffuse encephalopathy with greater degree of bifrontal cortical dysfunction. On day 4, the patient was weaned off all sedation with no further seizure-like activity, but still remained unable to follow commands. It was at this time the decision was made to discontinue metronidazole, vancomycin, and aztreonam as the patient had reached the predetermined treatment duration. The patient's serum creatinine was beginning to trend down as well from 4.0 to 3.0 to $2.6 \mathrm{mg} / \mathrm{dL}$ ( 353.6 to 265.2 to $229.8 \mu \mathrm{mol} / \mathrm{L}$ ). On day 5 , the patient was able to follow commands and was subsequently extubated, serum creatinine was down to $2.4 \mathrm{mg} / \mathrm{dL}(212.2$ $\mu \mathrm{mol} / \mathrm{L}$ ), all cultures were negative to date, and there was no repeated seizure activity since the initial insult. On day 8 , the patient was transferred to the general medicine ward. At no point during admission did the patient become febrile, require renal replacement therapy, or reach a systolic blood pressure $>160 \mathrm{~mm} \mathrm{Hg}$, which are known risk factors for the development of PRES. The patient continued to gradually regain strength and by day 15 was near-baseline strength, and placement to an acute rehabilitation facility on discharge was determined to be her safest course. This was arranged over the next 3 days. On day 18 of her admission, a subsequent MRI of the brain was performed, which showed complete resolution of the previously seen images suggestive of PRES. The patient completed an extensive admission with near-complete renal recovery, no further seizure activity, and was discharged back to the rehabilitation facility on day 18 . Ultimate diagnosis per the neurocritical and infectious diseases team was PRES induced by ciprofloxacin, metronidazole, or her concomitant renal failure. A Naranjo Adverse Drug Reaction Probability Scale was performed for both ciprofloxacin and metronidazole, which resulted in a score of 5 for both agents, which correlates to a probable reaction secondary to the medications.

\section{Discussion}

Currently, no consensus exists on the proposed pathogenesis of PRES. One mechanism postulated is that severe hypertension causes an interruption in cerebral autoregulatory processes. ${ }^{9}$ Uncontrolled hypertension can cause increased damage to cerebral vessels, resulting in release of interstitial contents. Another possible theory is that a dysregulation in inflammatory cytokines causes dysfunction in endothelial cells within the brain. Finally, a theory of a potential reversible vasospasm secondary to overactivity of cerebral autoregulatory function in high vascular border territories of the brain has been proposed as a possible mechanism of disease. ${ }^{10}$

The presentation of PRES can vary, but there are particular hallmark signs and symptoms that have been documented in the available literature. A variety of neurological symptoms are seen, including headaches, seizures, encephalopathy, tetanus, and visual changes. ${ }^{4,11}$ The current literature suggests that MRI is superior to CT in the identification of PRES. ${ }^{6}$ Typical PRES lesions are seen as hyper- to isointense on FLAIR/T2-weighted images seen on MRI. ${ }^{4}$ Wernicke's encephalopathy and PRES have overlapping MRI findings and symptoms, making the diagnosis difficult in some cases where a patient may have an extensive alcohol use history. 
Regarding medication-induced PRES, occurrence associated with immunosuppressive agents is the most well documented in the literature. Antibiotic-induced PRES is much less reported, and as PRES is a diagnosis of exclusion, the true incidence is hard to quantify. Currently, literature exists documenting linezolid and ciprofloxacin as potential antibiotics linked to PRES. In comparison, however, MIE has been well documented with 64 cases identified in a systematic review performed by Kuriyama et al. ${ }^{12}$ Onset of MIE usually occurs with a cumulative dose of 20 to $120 \mathrm{~g}$ of metronidazole over the course of 2 to 6 weeks; however, patients with renal dysfunction, hepatic failure, or the pediatric population could experience quicker onset. ${ }^{7}$ Differentiating between MIE and PRES can be difficult when the patient presents with signs and symptoms consistent with both disorders. ${ }^{3-7}$ Imaging was a major factor in determining PRES versus MIE in this patient, as symptomatically they present very similar symptoms. The patient had hyperintensity in the parietal occipital regions on MRI, which is indicative of PRES, whereas MIE is associated with lesions in a variety of areas, for example, the cerebellar dentate nucleus. ${ }^{13}$ At the time of our literature review, no case reports have identified metronidazole as an agent which has induced PRES.

The only prior case of ciprofloxacin-induced PRES involved a 16-year-old male who was being treated for a chest infection. $^{2}$ After 3 days of treatment, the patient developed a diffuse headache, dizziness, drowsiness, and dyspnea and finally developed generalized tonic-clonic seizures. During this development, initial CT was negative and cerebrospinal fluid showed no abnormalities. The patient was ultimately transferred to the intensive care unit and intubated for airway protection. On the fourth day of treatment, the patient had an MRI performed, which showed a T2 hyperintensity in the posterior cerebellar region and bilaterally in the occipital region. It was at this point the decision was made to discontinue ciprofloxacin and transition to cefepime. The patient was extubated 3 days after discontinuation of ciprofloxacin, with no residual signs of weakness or central nervous system effects. A repeat MRI 5 days after discontinuation showed no abnormalities and complete reversal of PRES. The author concluded that the PRES had been induced by ciprofloxacin as he had no other possible risk factors.

No current treatment for PRES exists; however, strategies in the management of these patients include discontinuation of the offending agent, possible seizure prophylaxis, and maintaining normotensive blood pressures. ${ }^{3}$ Early detection and prompt correction of the underlying causative factor decrease the risk of damage to the cerebral vascular system. ${ }^{6}$ As for this presented case, the patient was never hypertensive and upon presentation actually required vasopressor support. With respect to sepsis and inflammatory status, the patient was never febrile and had an initial leukocytosis which was considered reactive secondary to the patient seizing. It is plausible to consider the patient's renal function and chronic antibiotic use for this to be a pharmacotoxic case of antibiotic-induced PRES.

\section{Conclusion}

This case highlights the importance of antimicrobial selection with respect to agent, dose, and duration of therapy in elderly patients or those who require long-term treatment, as prolonged exposure to the antibiotic agents mentioned throughout this case is a clear risk factor in the development of PRES and MIE. It remains to be determined whether metronidazole, ciprofloxacin, or a combination of both was responsible for this case of PRES; however, the temporal relationship makes it probable that these agents were the culprits in this case.

\section{Declaration of Conflicting Interests}

The author(s) declared no potential conflicts of interest with respect to the research, authorship, and/or publication of this article.

\section{Funding}

The author(s) received no financial support for the research, authorship, and/or publication of this article.

\section{ORCID iD}

Brian Wesley Gilbert iD https://orcid.org/0000-0002-1385-7500

\section{References}

1. Hobson EV, Craven I, Blank SC. Posterior reversible encephalopathy syndrome: a truly treatable neurologic illness. Perit Dial Int. 2012;32(6):590-594.

2. Ali WH. Ciprofloxacin-associated posterior reversible encephalopathy. BMJ Case Rep. 2013;bcr2013008636.

3. Zappella N, Perier F, Pico F, et al. Duloxetine-related posterior reversible encephalopathy syndrome: a case report. Medicine (Baltimore). 2016;95(33):e4556.

4. Nagel S, Kohrmann M, Huttner HB, Storch-Hagenlocher B, Schwab S. Linezolid-induced posterior reversible leukoencephalopathy syndrome. Arch Neurol. 2007;64(5):746-748.

5. Zhang L, Xu J. Posterior reversible encephalopathy syndrome (PRES) attributed to mycophenolate mofetil during the management of SLE: a case report and review. Am J Clin Exp Immunol. 2018;7:1-7.

6. Kapoor A, Birks E, Lenneman A, McCants K. Posterior reversible encephalopathy syndrome after heart transplantation: diagnosis and immunosuppressive therapy. Tex Heart Inst J. 2017;44(3):205-208.

7. Godfrey MS, Finn A, Zainah H, Dapaah-Afriyie K. Metronidazole-induced encephalopathy after prolonged metronidazole course for treatment of C. BMJ Case Rep. 2015;1-3.

8. Dhar A, Manoila I, Dugay MH, Sellal F. Mystery case: metronidazole-induced encephalopathy. Neurology. 2016;87(9): e89-e90.

9. Bartynski W. Posterior reversible encephalopathy syndrome, part 2: controversies surrounding pathophysiology of 
vasogenic edema. AJNR Am JNeuroradiol. 2008;29(6):10431049.

10. Casey SO, Sampaio RC, Michel E, Truwit CL. Posterior reversible encephalopathy syndrome: utility of fluidattenuated inversion recovery MR imaging in the detection of cortical and subcortical lesions. AJNR Am J Neuroradiol. 2000;21(7):1199-1206

11. Kikuchi S, Orii F, Maemoto A, Ashida T. Reversible posterior leukoencephalopathy syndrome associated with treatment for acute exacerbation of ulcerative colitis. Intern Med 2016;55(5):473-477.

12. Kuriyama A, Jackson JL, Doi A, Kamiya T. Metronidazoleinduced central nervous system toxicity: a systematic review. Clin Neuropharmacol. 2011;34(6):241-247.

13. Kim E, Na DG, Kim EY, Kim JH, Son KR, Chang KH. MR Imaging of metronidazole-induced encephalopathy: lesion distribution and diffusion-weighted imaging findings. AJNR Am J Neuroradiol. 2007;28(9):1652-1658 